\title{
Estimation of three-dimensional water column sound speed profiles and sediment compressional wave speed and density profiles using a distributed network of buoys
}

Subramaniam D. Rajan, and George V. Frisk

Citation: The Journal of the Acoustical Society of America 147, 1392 (2020); doi: 10.1121/10.0000794

View online: https://doi.org/10.1121/10.0000794

View Table of Contents: https://asa.scitation.org/toc/jas/147/3

Published by the Acoustical Society of America

\section{ARTICLES YOU MAY BE INTERESTED IN}

Measurement and modeling of sound propagation over continental slope in the South China Sea

The Journal of the Acoustical Society of America 147, EL209 (2020); https://doi.org/10.1121/10.0000801

Multiple underwater sound scattering by cylindrically symmetric anomalies

The Journal of the Acoustical Society of America 147, 1429 (2020); https://doi.org/10.1121/10.0000827

Seasonally-invariant head wave speed extracted from ocean noise cross-correlation

The Journal of the Acoustical Society of America 147, EL241 (2020); https://doi.org/10.1121/10.0000877

Nonlinear time-warping made simple: A step-by-step tutorial on underwater acoustic modal separation with a single hydrophone

The Journal of the Acoustical Society of America 147, 1897 (2020); https://doi.org/10.1121/10.0000937

Time-warping in underwater acoustic waveguides

The Journal of the Acoustical Society of America 147, 898 (2020); https://doi.org/10.1121/10.0000693

The small-slope approximation for layered, fluid seafloors

The Journal of the Acoustical Society of America 147, 56 (2020); https://doi.org/10.1121/10.0000470

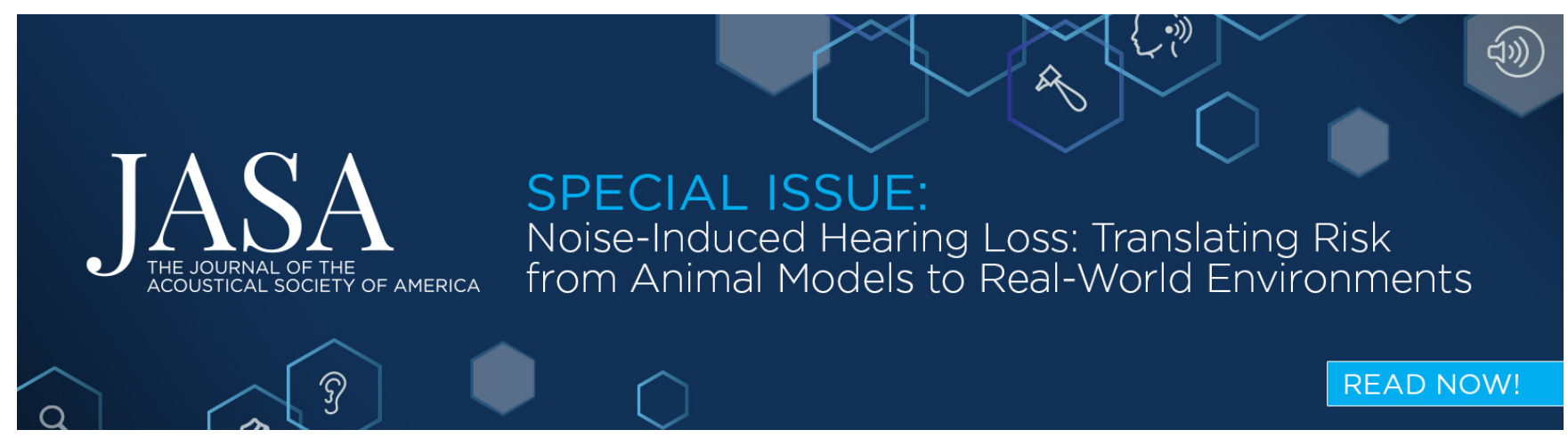




\title{
Estimation of three-dimensional water column sound speed profiles and sediment compressional wave speed and density profiles using a distributed network of buoys
}

Subramaniam D. Rajan ${ }^{1, a)}$ and George V. Frisk ${ }^{2, b)}$

${ }^{1}$ Scientific Solutions Inc., Nashua, New Hampshire 03063, USA

${ }^{2}$ Department of Ocean and Mechanical Engineering, Florida Atlantic University, Dania Beach, Florida 33004, USA

\begin{abstract}
:
Broadband data acquired during the Modal Mapping Experiment (MOMAX) V experiment are used to invert simultaneously for the three-dimensional (3D) water column sound speed profiles and the compressional wave speed and density profiles of the seabed in shallow waters off the coast of New Jersey. Linear Frequency Modulation sweep signals in the band $50-300 \mathrm{~Hz}$ are transmitted from a nearly stationary source at several discrete positions to a set of freely drifting receivers. Mode travel times are estimated from the signals acquired by the drifting buoys, and these are then used as input data in an inversion algorithm that estimates the acoustic properties of the water column and sediments. The resulting 3D compressional wave speed profiles in the seabed are generally consistent with the onedimensional profile obtained during the narrowband component of MOMAX V, as well as the results from other experiments in the same area. The validity of the inversion results has also been assessed by the ability of the inverted model to predict the fields measured during the narrowband experiments.
\end{abstract}

(C) 2020 Acoustical Society of America. https://doi.org/10.1121/10.0000794

(Received 2 October 2019; revised 23 January 2020; accepted 6 February 2020; published online 2 March 2020)

[Editor: D. Benjamin Reeder]

Pages: $1392-1403$

\section{INTRODUCTION}

The sediment acoustic properties in shallow water can be estimated using inversion methods that use modal data, such as modal eigenvalues or mode travel times, as input data. These methods have been demonstrated using data obtained in field experiments. ${ }^{1,2}$ Specifically, the Modal Mapping Experiments, termed MOMAX, generally use a distribution of freely drifting buoys, each with a hydrophone and Global Positioning System (GPS) receiver, to receive low-frequency tones transmitted by a moving source. Analysis of these data provides estimates of modal eigenvalues from which the geoacoustic properties of the sediment can be determined. The MOMAX V experiment, ${ }^{3}$ conducted in March 2011, had a broadband component as well. In this part of the experiment, the ship moved to discrete locations from which it broadcast broadband signals. The ship was nearly stationary during the transition of the broadband signals. These transmissions were received on a set of freely floating buoys. This configuration closely mimicked the operational scenario where sonobuoy receivers are deployed in a widely dispersed area in an Anti-submarine Warfare (ASW) operation, along with a sonobuoy that carries a source. The analysis presented in this paper demonstrates

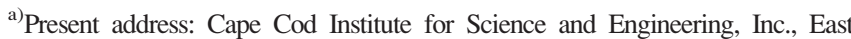
Sandwich, Massachusetts 02537, USA. Electronic mail: subra1933@gmail.com

b) Also at: Department of Applied Ocean Physics and Engineering, Woods Hole Oceanographic Institution, Woods Hole, Massachusetts 02543, USA, ORCID: 0000-0001-8480-6092.
}

the possibility of rapid estimation of geoacoustic properties in shallow water using data collected in such routine naval sorties.

In a previously published paper, ${ }^{4}$ the broadband data collected during this experiment were used to determine the variability in the sound speed profile of the water column with respect to space and time. The sediment compressional wave speed properties from previous inversions were used as known input data in performing the analysis. More detailed knowledge was not necessary as only the lower order modes from higher frequencies with limited bottom penetration were used in the analysis. In contrast, we present results of the analysis of the broadband data to estimate simultaneously the three-dimensional (3D) water column and sediment compressional wave speed and density profiles for the entire region. The data used in the analysis are the mode travel times in the frequency band of $50-110 \mathrm{~Hz}$.

A number of papers have been published where the mode dispersion data are used to estimate the sediment parameters. ${ }^{2,5-7}$ Different methodologies have been employed for estimating the sediment parameters from experimentally determined mode dispersion data. They vary from a linearized inverse procedure based on perturbation theory to full nonlinear inversion based on Bayesian theory. In the analysis presented in this paper, we use a linearized inversion procedure to estimate the water column and sediment properties.

The paper is organized as follows. In Sec. II, we provide a brief description of the inversion methodology and the experiment together with details of the data analysis. A 
discussion of the results is presented in Sec. III. Section IV summarizes the results of the analysis.

\section{INVERSION APPROACH, EXPERIMENT DESCRIPTION, AND DATA ANALYSIS}

\section{A. Inversion methodology}

Consider a range-independent ocean model whose compressional wave speed and density are represented by $c_{b}(z)$ and $\rho_{b}(z)$, respectively. For this model, $k_{n}$ and $\varphi_{n}$ are the eigenvalue and mode function of the $n$th mode, respectively, that satisfy the Helmholtz equation and boundary conditions associated with the waveguide model. We now perturb the compressional wave speed by a small quantity $\Delta c(z)$. This will result in a change in the group speeds of the propagating modes. It has been shown that the change in group speed of the $n$th mode, due to the perturbation of the compressional wave speed, is given by ${ }^{1}$

$$
\frac{1}{\gamma_{n}(\omega)}-\frac{1}{\hat{\gamma}_{n}(\omega)}=\frac{\partial}{\partial \omega} \int_{0}^{\infty} \frac{-1}{k_{n}(\omega)} \frac{\omega^{2} \Delta c(z)}{c_{b}^{3}(z) \rho_{b}(z)}\left|\varphi_{n}(z, \omega)\right|^{2} \mathrm{dz} .
$$

In the above equation, $\gamma_{n}$ and $\hat{\gamma}_{n}$ represent the group speeds of mode $n$ for the perturbed and unperturbed ocean models, respectively, $\omega$ is the frequency of the acoustic source, and $z$ is the depth below the sea surface.

The mode travel time is the range from the source to the receiver divided by the group speed of the mode in question, such that the term on the left-hand side of Eq. (1) is related to the perturbation in mode arrival time, and is given by

$$
d t_{n}=\frac{r}{\gamma_{n}(\omega)}-\frac{r}{\hat{\gamma}_{n}(\omega)},
$$

where $d t_{n}$ is the perturbation in mode travel time, and $r$ is the range to the receiver.

By discretizing the waveguide environment in depth, the integral in Eq. (1) can be approximated by a sum as given by

$$
\frac{1}{\gamma_{n}(\omega)}-\frac{1}{\hat{\gamma}_{n}(\omega)}=\sum_{q=1}^{Q} G_{n q}(\omega) \Delta c\left(z_{q}\right)
$$

This sum can be reduced to a matrix equation of the form $\boldsymbol{d}=\boldsymbol{G} \boldsymbol{m}$, where $\boldsymbol{d}$ is a vector containing the perturbation in modal travel times for different modes and at different frequencies, $G$ is a matrix whose elements are $G n q$, representing the background model, and $\boldsymbol{m}$ is a vector that contains $\Delta c(z)$, the perturbations to the compressional wave speed profile. The matrix equation is then inverted to determine the quantity $\Delta c\left(z_{q}\right), q=1, \ldots, Q$.

A procedure to jointly estimate the compressional wave speed and density profiles from modal eigenvalues has been proposed. ${ }^{8}$ It is now modified to estimate both the compressional wave speed and density profiles from mode dispersion data. This procedure is also a linearized solution based on perturbation theory. Consider a range-independent horizontally stratified ocean model with iso-velocity and iso-density layers. The compressional wave speed and density in the $m$ th layer are represented by $c_{\mathrm{bm}}$ and $\rho_{\mathrm{bm}}$, respectively. For this model, $k_{\mathrm{bn}}$ and $\varphi_{\mathrm{bn}}(z)$ are the eigenvalue and mode function of the $n$th mode, respectively. We now perturb the compressional wave speed and density of the $m$ th layer by small quantities $\Delta c_{m}$ and $\Delta \rho_{m}$. The resulting change in modal eigenvalue is given by ${ }^{8}$

$$
\begin{aligned}
\Delta k_{n}= & \frac{-\omega^{2} \Delta c_{m}}{c_{\mathrm{bm}}^{3} \rho_{\mathrm{bm}} k_{\mathrm{bn}}} \int_{z_{m}}^{z_{m+1}} \varphi_{\mathrm{bn}}^{2}(z) \mathrm{dz} \\
& +\left.\frac{\Delta \rho_{m}}{4 \rho_{\mathrm{bm}}^{2} k_{\mathrm{bn}}} \frac{d}{\mathrm{dz}} \varphi_{\mathrm{bn}}^{2}(z)\right|_{z_{m}} ^{z_{m+1}}
\end{aligned}
$$

Equation (4) can be extended to a case when changes occur in all of the layers. By taking the derivate of Eq. (4) with respect to frequency, the changes in eigenvalues, as presented in the left-hand side of Eq. (4), can be modified to changes in mode group speed. The resulting expression is

$$
\begin{aligned}
\frac{1}{\gamma_{n}(\omega)}-\frac{1}{\hat{\gamma}_{n}(\omega)}= & \frac{d}{\mathrm{~d} \omega}\left\{\sum_{m=1}^{m=M} \frac{-\omega^{2} \Delta c_{m}}{c_{\mathrm{bm}}^{3} \rho_{\mathrm{bm}} k_{\mathrm{bn}}} \int_{z_{m}}^{z_{m+1}} \varphi_{\mathrm{bn}}^{2}(z) \mathrm{dz}\right\} \\
+ & \frac{d}{\mathrm{~d} \omega}\left\{\left.\sum_{m=1}^{m=M} \frac{\Delta \rho_{m}}{4 \rho_{\mathrm{bm}}^{2} k_{\mathrm{bn}}} \frac{d}{\mathrm{dz}}\left[\varphi_{\mathrm{bn}}^{2}\right]\right|_{z_{m}} ^{z_{m+1}}\right\} \\
& n=1, \ldots, N
\end{aligned}
$$

In Eq. (5), $\gamma_{n}$ and $\hat{\gamma}_{n}$ represent the group speeds of mode $n$ for the unperturbed and perturbed ocean models, respectively. Equation (5) is then reduced to a matrix equation of the form

$$
\Delta \boldsymbol{\gamma}=\{\boldsymbol{A} \mid \boldsymbol{B}\}\left[\begin{array}{c}
\Delta \boldsymbol{P}_{1} \\
\vdots \\
\Delta \boldsymbol{P}_{2 M}
\end{array}\right],
$$

where the vector $\left[\Delta \boldsymbol{P}_{1} \cdots \Delta \boldsymbol{P}_{2 \boldsymbol{M}}\right]^{\boldsymbol{T}}$ represents the corrections to the compressional wave speed and density of the $M$ layers.

The elements of the matrices $\boldsymbol{A}$ and $\boldsymbol{B}$ and the vector $\Delta \gamma$ are

$$
\begin{aligned}
& A_{\mathrm{nk}}=\frac{d}{\mathrm{~d} \omega}\left\{\frac{-\omega^{2} \Delta c_{k}}{c_{\mathrm{bk}}^{3} \rho_{\mathrm{bk}} k_{\mathrm{bn}}} \int_{z_{k}}^{z_{k+1}} \varphi_{\mathrm{bn}}^{2}(z) \mathrm{dz}\right\}, \\
& B_{\mathrm{nm}}=\frac{d}{\mathrm{~d} \omega}\left\{\left.\frac{\Delta \rho_{m}}{4 \rho_{\mathrm{bm}}^{2} k_{\mathrm{bn}}} \frac{d}{\mathrm{dz}}\left[\varphi_{\mathrm{bn}}^{2}(z)\right]\right|_{z_{m}} ^{z_{m+1}}\right\}, \\
& \Delta \gamma_{n}=\frac{1}{\gamma_{n}(\omega)}-\frac{1}{\hat{\gamma}_{n}(\omega)}, \quad n=1, \ldots, N .
\end{aligned}
$$

The matrix equation in Eq. (6) is generally ill-conditioned, and special care should be taken to solve it. A means of regularization of the solution is required to obtain meaningful solutions to the matrix equation.

In the case of a range-dependent environment, the modal travel time perturbation for the $n$th mode caused by changes in the compressional wave speed is given by ${ }^{2}$ 


$$
d t_{n}=\frac{\partial}{\partial \omega} \int_{0}^{r} \int_{0}^{\infty} \frac{-1}{k_{n}(s, \omega)} \frac{\omega^{2} \Delta c(s, z)}{c_{b}^{3}(s, z) \rho_{b}(s, z)}\left|\varphi_{n}(s, z, \omega)\right|^{2} d s d z .
$$

For a given source/receiver geometry, we divide the total range $R$ between the source and receiver into $P$ rangeindependent segments. The water column and sediment properties are a function of depth only in each rangeindependent section. By discretizing the environment in both range and depth, the double integral can be changed into a double sum as given by

$$
d t_{n, \omega}=\sum_{p=1}^{P} \sum_{q=1}^{Q} G_{\mathrm{npq}}\left(\omega, s_{p}, z_{q}\right) \Delta c\left(s_{p}, z_{q}\right) .
$$

If $\gamma_{n, \omega}(p)$ is the group velocity of mode $n$ at frequency $\omega$ in section $p$ for the perturbed ocean model, and $\hat{\gamma}_{n, \omega}(p)$ is the group velocity of mode $n$ at frequency $\omega$ in section $p$ for the unperturbed ocean model, the travel time difference $d t_{n, \omega}$ is given by

$$
d t_{n, \omega}=\sum_{p=1}^{P} \frac{r_{p}}{\gamma_{n, \omega}(p)}-\sum_{p=1}^{P} \frac{r_{p}}{\hat{\gamma}_{n, \omega}(p)} .
$$

In a field experiment, the experimentally determined mode travel time will be the first term on the right-hand side of Eq. (10), and the second term is the sum of the mode travel times along the different sections as they travel from source to receiver, and these are determined by the background model for each section.

As was done previously, considering the set of linear equations in Eq. (9) for all modes $n$ and frequencies $\omega$, this double sum can be reduced to a matrix equation that is solved to determine the quantity $\Delta c\left(s_{p}, z_{q}\right), p=1, \ldots, P, q=1, \ldots, Q$, where $s_{p}$ refers to the $p$ th step in the range, and $z_{q}$ refers to the $q$ th step in the depth. Similar extension to deal with rangedependent scenarios can also be made when jointly estimating the compressional wave speed profile and density profile. Equation (9) is then put in the form of a matrix equation $\boldsymbol{G m}=\boldsymbol{d}$ and solved for $\boldsymbol{m}$, which represents the corrections to the water column sound speed, the sediment compressional wave speed, and the sediment density in each range interval.

In order to solve this range-dependent problem, a multiplicity of source/receiver combinations is necessary. ${ }^{2}$ By using a multiplicity of sources and receivers, a set of signals corresponding to each source/receiver combination is generated. Time-frequency analysis of the received signals gives an estimate of the arrival times for each mode at different frequencies along the paths of each source/receiver combination. These data are the input to the inversion algorithm.

The matrix equation to be solved is generally illconditioned and requires some form of regularization to obtain stable, meaningful solutions. Qualitative regularization $(\mathrm{QR})^{9,10}$ was chosen to solve the discrete inverse problem. QR is an extension of Tikhonov regularization, ${ }^{11}$ an approach that places a constraint on derivatives of the solution that ensures smoothness. QR allows for piecewise smooth solutions and can incorporate a priori information to represent layered media. When this constraint is included, the solution is found by satisfying both the data and the constraint. In QR, the following equations must be satisfied:

$$
\begin{aligned}
& G m=d, \\
& L_{q}=\mathbf{0} .
\end{aligned}
$$

The operator $\boldsymbol{L}_{q}$ is defined by

$$
\boldsymbol{L}_{q}=\boldsymbol{L}\left(\boldsymbol{I}-\sum_{i=1}^{r} \boldsymbol{q}_{i} \boldsymbol{q}_{i}^{T}\right)
$$

where $\boldsymbol{L}$ is a discrete version of the differential operator $d^{n} / d z^{n}$, as used in Tikhonov regularization, and the set of vectors $\left\{\mathbf{q}_{i}\right\}_{i=1}^{r}$ is an orthogonal basis for the subspace $Q$ that contains all models that have discontinuities at the prescribed locations. For $n=2$, the differential operator results in a piecewise smooth solution. The least square solution to Eq. (11) is given by

$$
\hat{\boldsymbol{m}}=\left(G^{T} G+\lambda^{2} L_{q}^{T} L_{q}\right)^{-1} G^{T} d,
$$

where $\hat{\boldsymbol{m}}$ is the solution, $\lambda$ is a Lagrange multiplier, and $T$ represents a transpose.

When inverting for water column sound speed, as well as sediment properties, additional constraints are necessary. ${ }^{12}$ These constraints prevent any changes at prescribed locations while performing inversions. To obtain stable and reliable estimates for the water column sound speed, the initial assumed values for the water column sound speed close to the ocean surface and bottom are restricted and remain unchanged by the inversion process. With this additional constraint, known as the approximate equality constraint, the solution is one that satisfies the data and the constraints

$$
\begin{aligned}
& G m=d, \\
& L_{q}=0, \\
& A m=\alpha .
\end{aligned}
$$

The matrix $\boldsymbol{A}$ is a matrix that identifies the location where the approximate equality constraint is applied, and $\alpha$ is a vector of zeros. The least squares solution in this case is

$$
\hat{\boldsymbol{m}}=\left(G^{T} G+\lambda_{1} L_{q}^{T} L_{q}+\lambda_{2} A^{T} A\right)^{-1} G^{T} d .
$$

The quantities $\lambda_{1}$ and $\lambda_{2}$ are Lagrange multipliers chosen to achieve a stable solution.

In order to estimate the water column sound speed profiles, sediment compressional wave speed profiles, and density profiles, we assume that we have approximate estimates of these profiles from archival data or some other source. These estimates of the profiles are then used as the initial background models for the inversion algorithm. The true 
water column sound speed and sediment compressional wave speed profiles are assumed to be small perturbations to these initial estimates of the profiles.

Taking the modal group speeds estimated from the data and calculated for the background model, the perturbation $\Delta c(s, z)$ and $\Delta \boldsymbol{\rho}(\boldsymbol{s}, \boldsymbol{z})$ are obtained by inverting Eq. (9). The perturbation profile is added to the initial background model to obtain the true model. In practice, the inversion is carried out iteratively with the background model updated by $\Delta c(s, z)$ and $\Delta \rho(s, z)$ after each inversion calculation. After several iterations, the difference between group velocities estimated from field data and the updated models approaches zero. These updated models are taken as the result of the inversion procedure.

The reliability of the inverse results is assessed by estimating the covariance of the estimated model parameters. To obtain the covariance of the model parameters, we follow the procedure outlined in Ref. 13. The basic equation for obtaining a solution of model parameters is of the form $\boldsymbol{m}=\boldsymbol{G}^{-1} \boldsymbol{d}$. For an error $\Delta \boldsymbol{d}$ in the data, the error in the solution of model parameters will be $\Delta \boldsymbol{m}=\boldsymbol{G}^{-1} \Delta \boldsymbol{d}$. The variance of the model parameters $C_{m}$ is then given by

$$
\begin{aligned}
& \left\langle\left(\Delta m \Delta m^{T}\right)\right\rangle=G^{-1}\left\langle\Delta d \Delta d^{T}\right\rangle\left(G^{-1}\right)^{T}, \\
& C_{m}=G^{-1} C_{d}\left(G^{-1}\right)^{T}
\end{aligned}
$$

The data covariance is estimated from the data acquired by the sonobuoys. In Eqs. (16) and (17), $\boldsymbol{T}$ represents the transpose of the matrix. The diagonal of the model covariance matrix provides an estimate of the variance of the model parameters, and the square root of the variance gives the standard deviation of the parameter. Note that the inverse of $\boldsymbol{G}$ is not generally obtainable as this is, in most cases, not a square matrix. Instead, $\boldsymbol{m}=\left(\boldsymbol{G}^{\boldsymbol{T}} \boldsymbol{G}\right)^{-1} \boldsymbol{G}^{\boldsymbol{T}} \boldsymbol{d}$ is used.

Another factor in the evaluation of the results of the inversion is the resolution of the estimates. The parameters we are attempting to obtain by the inverse methods are points on a continuous function, and therefore the parameter space has infinite dimension. However, the data we have to reconstruct the unknown function are available only on a finite set. Thus, the model obtained by the inverse cannot resolve all the features of the true model, and a smoothed out version of the model is obtained.

The estimate of the model parameters is given by $\boldsymbol{m}_{\mathrm{est}}=\boldsymbol{G}^{-1} \boldsymbol{d}$. Replacing the data vector by $\boldsymbol{G} \boldsymbol{m}$, we have $\boldsymbol{m}_{\mathrm{est}}=\boldsymbol{G}^{-1} \boldsymbol{G} \boldsymbol{m}$. If $\boldsymbol{R}=\boldsymbol{G}^{-1} \boldsymbol{G}$ is an identity matrix, then the estimate of the model parameters is the true value. If otherwise, the estimate of the model parameters is an averaged value. The averaging is based on the row vectors of the matrix $\boldsymbol{R}$. Another expression used for obtaining the averaging matrix ${ }^{12} \boldsymbol{R}$ is $\boldsymbol{G}^{\boldsymbol{T}}\left(\boldsymbol{G} \boldsymbol{G}^{\boldsymbol{T}}\right)^{-1} \boldsymbol{G}$. To quantify the resolution we compute the resolution length, a measure of the range over which averaging is done to obtain the estimate. One approach to obtain the resolution length $(r l)$ at each sediment layer depth $(n)$ is to use the expression ${ }^{12}$

$$
r l(n)=\frac{\sum_{1}^{M} R(n, m)^{2} d z}{R(n, n)^{2}},
$$

where $\boldsymbol{R}$ is a $(M \times M)$ matrix and $M$ represents the number of layers. If the averaging matrix is an identity matrix, then the resolution length will be $d z$, i.e., the discretization used in solving the inverse problem. Higher values of resolution length indicate that the solution is averaged over a greater range.

\section{B. Description of experiment}

The complete details of MOMAX $\mathrm{V}$ are described in Frisk et al., ${ }^{3}$ which analyzed the narrowband data collected during the experiment. The experiment included a broadband component in which the research vessel (R/V) Sharp transmitted broadband signals from several discrete locations. At these locations, the ship was nearly stationary. Three freely drifting MOMAX buoys, named Curly, Larry, and Moe, were deployed before the start of the broadband experiment. The locations of the ship and the buoys are shown in Fig. 1. The ship had a J15 source suspended at a nominal depth of $56 \mathrm{~m}$. The signal transmitted was an Linear Frequency Modulation sweep from $50 \mathrm{~Hz}$ to $300 \mathrm{~Hz}$. The duration of each ping was $0.5 \mathrm{~s}$. The pings were repeated every $3 \mathrm{~s}$ for a total duration of $3.5 \mathrm{~min}$ from each ship location. The MOMAX buoys had two hydrophones at nominal depths of $61 \mathrm{~m}$ and $64 \mathrm{~m}$. It is, however, mentioned that source and receiver depths are not required for the estimation of mode arrival times, although the location of the source and receiver will determine the modes that are excited. Data from 21 consecutive transmissions from each of the waypoints are summed up in order to improve the signal-to-noise ratio (SNR) and average out signal variations due to movement of the ship during transmission of the signal. Time synchronization was achieved by recording the transmitted signal on a hydrophone attached to the source

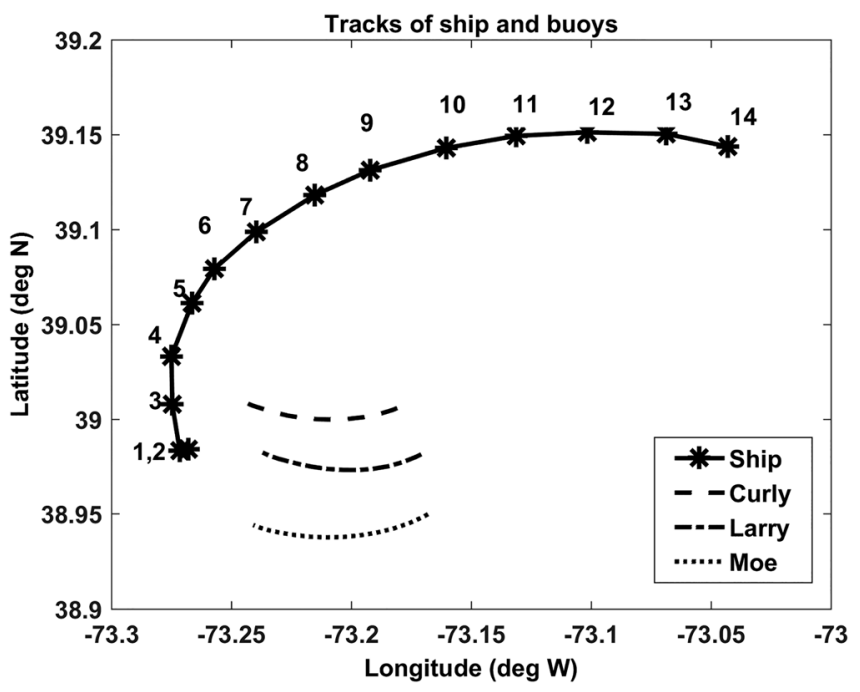

FIG. 1. The tracks of the ship and the buoys during the broadband experiment. The stars represent waypoints (numbered 1-14) at which the ship was nearly stationary. 
frame. There were 14 waypoints at which the ship was nearly stationary, and broadband signals were transmitted. The signals received by Curly at one of its hydrophones were used in the analysis. The signals received from transmissions at waypoints 1-10 were used in the analysis. Transmissions from waypoints 11 to14 have low SNR due to large distances between the source and receivers and, hence, were not included in the analysis. Before processing the signal acquired by the buoys for time-frequency analysis, it is necessary to extract the impulse response of the channel in order to take out the time-frequency variation of the transmitted signal during the signal duration. This was done by performing frequency domain deconvolution of the received signal.

The distances between the ship and the buoys were obtained using the data from GPS units mounted on the ship and the buoys. The bathymetry between the ship and the buoys was obtained from archival data available for the region. Based on differences in bathymetry, the region between the ship and buoy locations is divided into five regions, as shown in Fig. 2. The depths of the water column in regions I, II, III, IV, and V have an average value of $74 \mathrm{~m}, 70 \mathrm{~m}, 69 \mathrm{~m}, 69 \mathrm{~m}$, and $69 \mathrm{~m}$, respectively. Data used in the inversion are the mode travel times at frequencies $50-110 \mathrm{~Hz}$ in $10 \mathrm{~Hz}$ steps.

The signals for waypoints $1-10$ are analyzed using the warping method ${ }^{14}$ to estimate mode travel times. The warping method involves applying a warping function to the received signal. If $g$ is a function of $x$ and the warping operator is $h(x)$, the warped function $W_{g}(x)$ is

$$
W_{g}(x)=\sqrt{\frac{d h}{d x}} g(h(x)) .
$$

The warping function applied in our analysis of the signal received by the buoy is

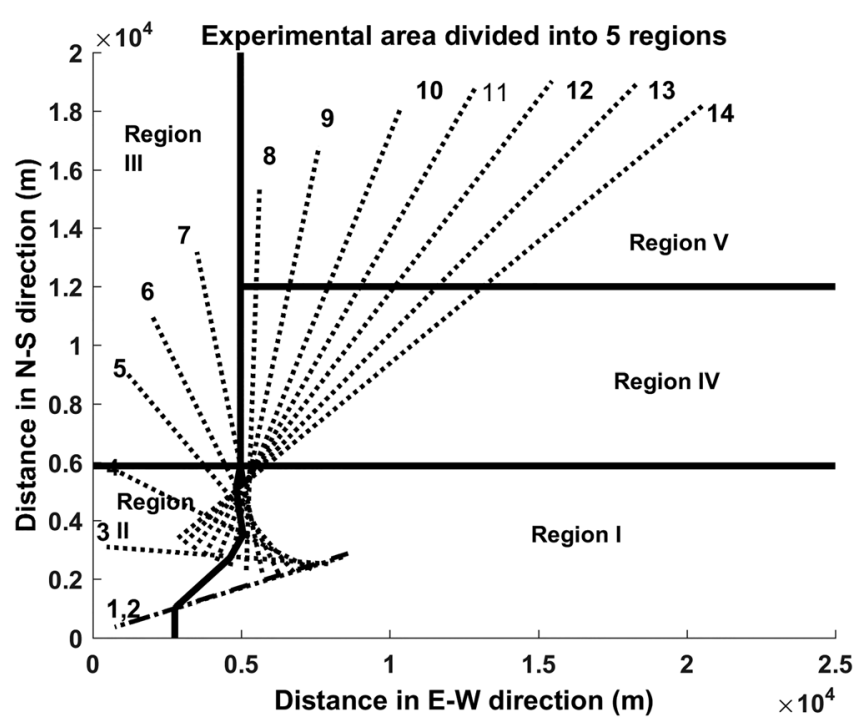

FIG. 2. The five regions into which the area is divided, and for which the water column sound speed profiles and sediment compressional wave speeds and density profiles are estimated. Dashed lines indicate sourcereceiver paths together with waypoint numbers.

$$
h(t)=\sqrt{t^{2}+\left(\frac{r}{c}\right)^{2}}
$$

where $r$ is the distance between the source and receiver, and $c$ is the sound speed in the water column. It has been shown that the accuracy of the quantity $r / c$ is not critical, and even an approximate value is adequate. ${ }^{15}$ Any warped function can be unwarped by applying the operator $h^{-1}(t)=\sqrt{t^{2}-(r / c)^{2}}$.

The estimation of the group speeds by processing the received signal $s(t)$ using the warping method is accomplished in the following stages:

(1) Use the warping operator to determine the warped signal $s_{\text {warped }}(t)$.

(2) Perform Short-time Fourier Transform (STFT) of the warped signal and obtain $S_{\text {warped }}(t, f)$.

(3) In this time frequency representation, the modes are resolved and well separated. Each mode can now be filtered.

(4) Perform inverse STFT on the filtered warped mode and obtain the warped mode $m d_{\text {warped }}(t)$.

(5) Unwarp the function $m d_{\text {warped }}(t)$ to get $m d(t)$.

(6) Perform STFT of $m d(t)$ to obtain the mode dispersion curve for the modes.

Measurements of ocean temperature were made using expendable bathythermograph (XBT) probes during the course of the experiment. The temperature profiles from XBT measurements together with the values of salinity obtained from conductivity-temperature-depth (CTD) measurements made during the narrowband experiment provided the information necessary to obtain the initial sound speed profile for the water column at each location of the XBT.

\section{Analysis of data}

The mode dispersion data estimated from the signals acquired by the various MOMAX buoys distributed over the area are now used to extract the acoustic characteristics of the water column and the sediments. This involves estimating the following parameters:

(1) The sound speed profile of the water column,

(2) the compressional wave speed and density of the sediment layers and the terminating half space, and

(3) the total thickness of the sediment and the layering information.

The quantities under items (1) and (2) above are determined using the inverse procedure outlined in Sec. II A. To carry out these inversions information on the layering of the sediment is required. The first step in the determination of the layering is to estimate the total thickness of the sediment. In order to determine the total thickness of the sediment layer, we assumed different values for the sediment thickness and for each of these values of sediment thickness inversion for the compressional wave speed in the layer was 

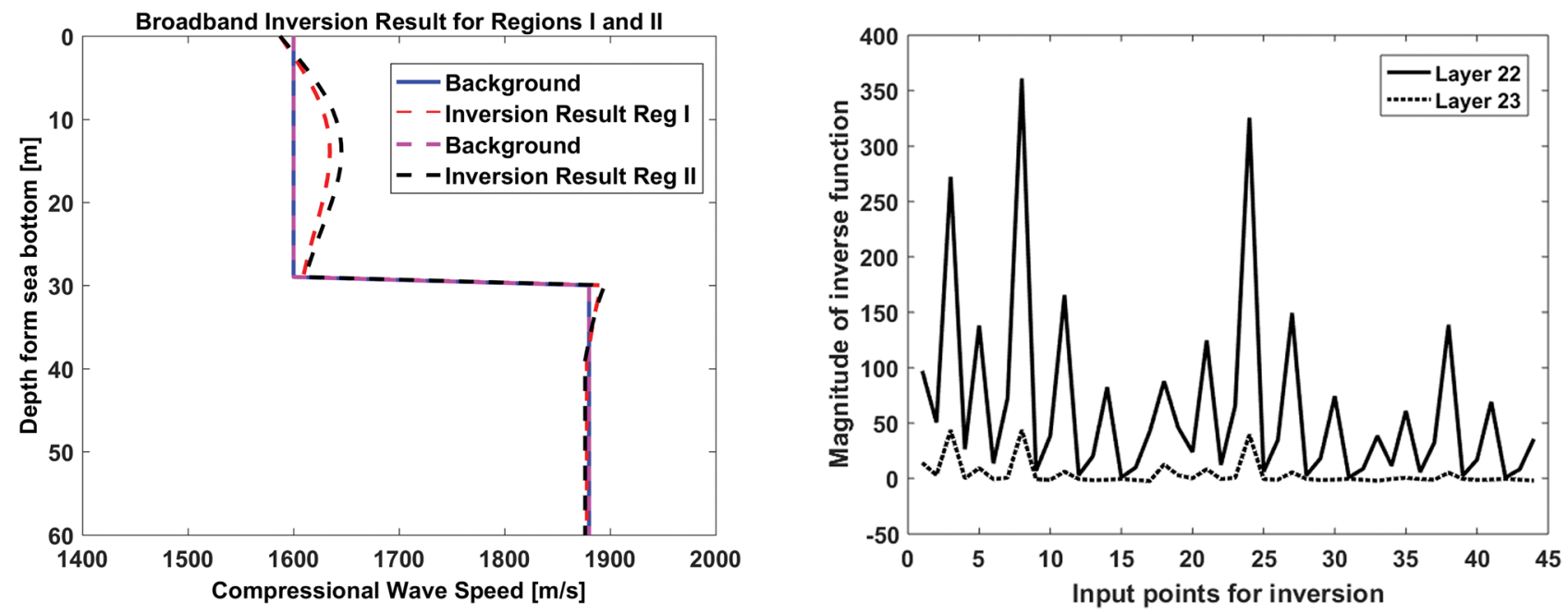

FIG. 3. (Color online) The left panel shows the inversion results for determining total layer thickness. This result is for regions I and II. The right panel shows the variation in the magnitude of the inverse function used in the estimate of the variance for layers 22 and 23 . The large reduction in the magnitude of the inverse function results in much smaller variance estimates for the deeper layers.

made. For the other unknowns in the inversion, namely the water column sound speed and density of the sediment layer, approximate values were assumed. The water column sound speed structure as determined during the experiment showed a well-mixed water column with almost uniform sound speed throughout the water column, and this value was used in the inversions. Note that in using an approximate value for the density in the sediment layer, we were guided by the fact that in earlier analyses of both broadband data $^{2}$ and narrowband data $^{3}$ an approximate value of the density of the sediment layers had been assumed, and compressional wave speed profiles for the sediment layers were estimated. This was possible because density values are less sensitive to inversions for compressional wave speed. This was the rationale for using an approximate value for density in our procedure for estimating the sediment layering. Further, in this inversion, qualitative regularization with identified layer depths was not used. Instead, inversion with regularization that provided a smooth solution for the variability of the compressional wave speed in the layer was used.

For each assumed sediment layer thickness, the inversion procedure produced a bottom model. The mean squared error between the mode data as predicted by the inverted model and the mode data used as input to the inversion was computed. The layer thickness that yields the minimum mean squared error is assumed to be the layer thickness of the sediment layers. The left panel of Fig. 3 shows the compressional wave speed profile as obtained in the inversion for regions I and II. Figure 3 indicates the presence of three layers: a low speed layer, a high speed layer, and a low speed layer terminated by a half space. Similar results were obtained in respect of regions III, IV, and V and, hence, layering as composed of three layers was adopted for all of the five regions. Having determined the total layer thickness and the number of layers, the procedure for determining the thickness of each layer was executed. Several combinations of layer thicknesses for the three layers, with the total thickness equal to the sediment thickness already estimated, were assumed and inversions performed for the compressional wave speed in the three layers using qualitative regularization. For each combination of layer thicknesses, we determine the mean squared error between the modal values predicted for the inverted model and the values used as input to the inversion process. The layer thickness model that yields the least mean squared error is chosen as the correct solution.

The seabed in the New Jersey shelf has been studied by a number of investigators. ${ }^{16,17}$ A large area of the shelf was also surveyed by Compressed High-Intensity Radiated Pulse (CHIRP) sonar and other means during the Shallow Water Experiment 2006 (SW06). ${ }^{18}$ A dominant feature of sediment structure as revealed by the survey is the " $R$ " reflector. The

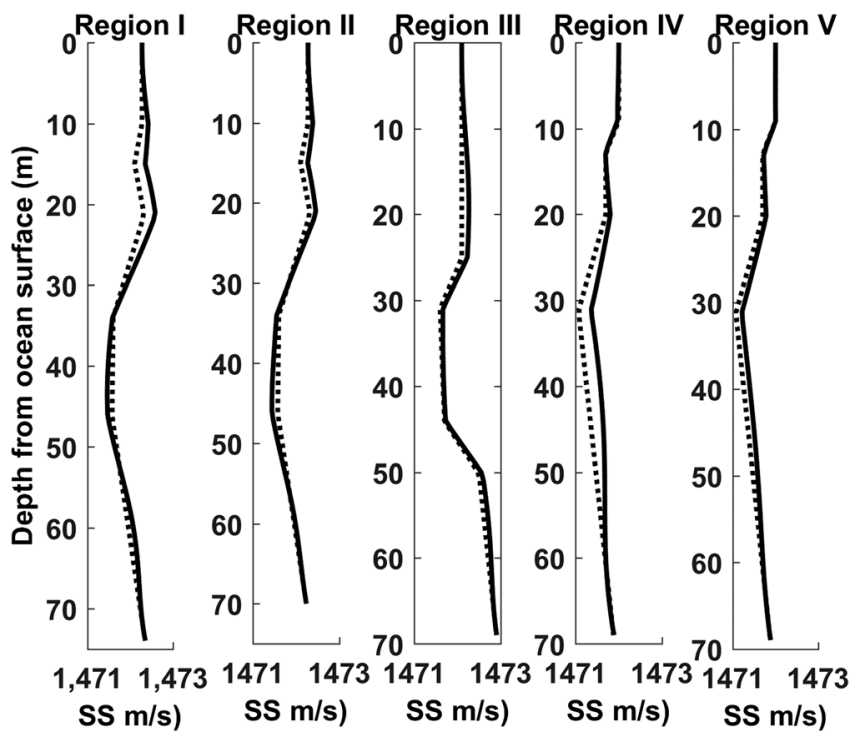

FIG. 4. The inverted water column sound speed profiles (solid lines) for the five regions, along with the initial background profiles (dotted lines). 
TABLE I. Compressional wave speed estimates (m/s).

\begin{tabular}{|c|c|c|c|c|c|c|c|}
\hline Region & $\begin{array}{c}\text { Layer } 1 \\
\text { thickness (m) }\end{array}$ & $\begin{array}{l}\text { Layer } 1 \text { wave } \\
\text { speed }(\mathrm{m} / \mathrm{s})\end{array}$ & $\begin{array}{c}\text { Layer } 2 \\
\text { thickness (m) }\end{array}$ & $\begin{array}{l}\text { Layer } 2 \text { wave } \\
\text { speed }(\mathrm{m} / \mathrm{s})\end{array}$ & $\begin{array}{c}\text { Layer } 3 \\
\text { thickness (m) }\end{array}$ & $\begin{array}{c}\text { Layer } 3 \text { wave } \\
\text { speed }(\mathrm{m} / \mathrm{s})\end{array}$ & $\begin{array}{c}\text { Half space wave } \\
\text { speed }(\mathrm{m} / \mathrm{s})\end{array}$ \\
\hline Region I & 6 & 1549 & 14 & 1644 & 9 & 1604 & 1856 \\
\hline Region II & 6 & 1594 & 14 & 1661 & 9 & 1609 & 1863 \\
\hline Region III & 6 & 1579 & 14 & 1687 & 10 & 1624 & 1887 \\
\hline Region IV & 6 & 1533 & 16 & 1638 & 10 & 1592 & 1870 \\
\hline Region V & 6 & 1506 & 16 & 1623 & 10 & 1593 & 1872 \\
\hline
\end{tabular}

reflector is covered by different layers of sediment. These are termed the diffuse outer layer and the inner layer unit. In some areas, another layer, termed erose boundary, is sandwiched between the layered units. In analyzing data collected during the Shallow Water Experiment 2006, this bottom sediment structure was used to interpret the results. Unfortunately, the area covered by the MOMAX V experiment does not fall within the area covered by the surveys. Extrapolating the data from the survey indicates that the inner layered unit is just beneath the ocean bottom, and the outer layer and erose boundary are not present. ${ }^{19}$ The thickness of the inner layered unit at the location of the MOMAX V experiment cannot be estimated in the absence of survey data. The layering structure of the sediments as estimated using the scheme described earlier, however, shows a $6 \mathrm{~m}$ thick inner layered unit in all of the five regions. The sediment layer thicknesses in the different regions as estimated are $6 \mathrm{~m}, 14 \mathrm{~m}$, and $9 \mathrm{~m}$ for regions I and II, $6 \mathrm{~m}, 14 \mathrm{~m}$, and $10 \mathrm{~m}$ for region III, and $6 \mathrm{~m}, 16 \mathrm{~m}$, and $10 \mathrm{~m}$ for regions IV and $\mathrm{V}$.

The initial sound speed profiles used to invert for the water column sound speed profiles were computed using the temperatures vs depth values obtained from the XBT data collected at locations close to waypoints $1,4,6$, and 8 and with the salinity value of 33.25 ppt. This value of salinity is based on the spread of salinity values recorded by CTDs deployed in the same general area during the narrowband component of the experiment. While inverting for the sound speed values in regions I and II, a mean profile based on XBT values at waypoints 1 and 4 was used. In the case of region III, the initial profile was based on the XBT data from waypoint 6 . In the case of regions IV and $\mathrm{V}$, the initial sound speed profile used for inversion was based on XBT data from waypoint 8 . The initial profiles and the inverted profiles are shown in Fig. 4.

The sediment compressional wave speeds and densities for the layers as determined by the inversion are listed in Tables I and II. The values for the three layers are close to one another for regions I, II, and III. In the case of regions IV and $\mathrm{V}$, we see that the compressional wave speed values in the top two layers (layers 1 and 2 in Table I) are much lower than the values for the top two layers in the other three regions. This behavior indicates that the sediment properties along the paths between source and receiver for waypoints 8,9 , and 10 are different from the sediment properties along the paths from waypoints 1 to 7 .

In the case of the inversions for the sound speed profiles in the water column, the mean standard deviations in the estimates of the sound speed $(\mathrm{m} / \mathrm{s})$ were $0.05,0.03,0.02$, 0.035 , and 0.02 for regions I, II, III, IV, and V, respectively. The mean resolution lengths $(\mathrm{m})$ of the water column sound speed for the regions I, II, III, IV, and V are 2.36, 2.29, 1.66, 2.59 , and 2.66, respectively. The deviation of the compressional wave speed and the density in all of the five regions are given in Tables III and IV. In order to obtain the data covariance matrix, individual pings from the source to the receiver were analyzed, and the mode travel times for the modes at the various frequencies were estimated. The data covariance matrix was estimated from the variability in the modal travel time estimated for individual pings. The model covariance matrix was estimated using Eq. (17). The standard deviation is the square root of the variance. Deviation equal to one standard deviation averaged over the thickness of each layer is the quantity in Tables III and IV. The deviation for layer 3 is much smaller than at other depths with respect to regions III, IV, and V. A possible cause for this behavior is that inversions were accomplished with data from only a reduced number of modes along one or more paths from source to receiver. In some instances, the third mode is not detectable in the lower frequencies and hence not included in the inversion. The fourth mode is not identifiable and hence not included in the inversion. As shown in Eq. (17), the variance of the estimates is determined by the structure of the quantity $\left(G^{T} G\right)^{-1} G^{T}$. In the right panel of Fig. 3 we plot the values of this quantity, corresponding to

TABLE II. Density estimates (gm/cc).

\begin{tabular}{lcccccc}
\hline \hline $\begin{array}{l}\text { Layer 1 } \\
\text { Region }\end{array}$ & $\begin{array}{c}\text { Layer 1 } \\
\text { thickness (m) }\end{array}$ & $\begin{array}{c}\text { Layer 2 } \\
\text { thickness (m) }\end{array}$ & $\begin{array}{c}\text { Layer 2 } \\
\text { density (gm/cc) }\end{array}$ & $\begin{array}{c}\text { Layer 3 } \\
\text { thickness (m) }\end{array}$ & $\begin{array}{c}\text { Layer 3 } \\
\text { density (gm/cc) }\end{array}$ & $\begin{array}{c}\text { Half space } \\
\text { density (gm/cc) }\end{array}$ \\
\hline Region I & 6 & 1.602 & 14 & 1.727 & 9 & 1.612 \\
Region II & 6 & 1.520 & 14 & 1.638 & 9 & 1.600 \\
Region III & 6 & 1.681 & 14 & 1.694 & 10 & 1.604 \\
Region IV & 6 & 1.786 & 16 & 1.704 & 10 & 1.596 \\
Region V & 6 & 1.549 & 16 & 1.708 & 10 & 1.597 \\
\hline \hline
\end{tabular}


TABLE III. Compressional wave speed deviations (Dev) and resolution lengths (RL) in the sediment for the five regions.

\begin{tabular}{|c|c|c|c|c|c|c|c|c|c|c|}
\hline \multirow[b]{2}{*}{ Layer } & \multicolumn{2}{|c|}{ Region I } & \multicolumn{2}{|c|}{ Region II } & \multicolumn{2}{|c|}{ Region III } & \multicolumn{2}{|c|}{ Region IV } & \multicolumn{2}{|c|}{ Region V } \\
\hline & $\operatorname{Dev}(\mathrm{m} / \mathrm{s})$ & $\mathrm{RL}(\mathrm{m})$ & $\operatorname{Dev}(\mathrm{m} / \mathrm{s})$ & $\mathrm{RL}(\mathrm{m})$ & $\operatorname{Dev}(\mathrm{m} / \mathrm{s})$ & $\mathrm{RL}(\mathrm{m})$ & $\operatorname{Dev}(\mathrm{m} / \mathrm{s})$ & $\mathrm{RL}(\mathrm{m})$ & $\operatorname{Dev}(\mathrm{m} / \mathrm{s})$ & $\mathrm{RL}(\mathrm{m})$ \\
\hline 1 & 12.70 & 1.017 & 13.28 & 1.049 & 14.08 & 1.085 & 8.156 & 1.097 & 4.921 & 1.144 \\
\hline 2 & 4.74 & 2.764 & 6.22 & 2.673 & 12.83 & 2.851 & 12.557 & 4.242 & 14.90 & 4.599 \\
\hline 3 & 7.12 & 5.455 & 6.69 & 5.302 & 3.01 & 5.838 & 1.453 & 7.411 & 1.82 & 7.524 \\
\hline
\end{tabular}

layers at depths $22 \mathrm{~m}$ and $23 \mathrm{~m}$ for region $\mathrm{V}$. Notice that the values are considerably lower in the case of the layer at a depth of $23 \mathrm{~m}$. This behavior is due to the diminished values of the mode function for these layer depths, which causes the considerable reduction in the estimate of the deviation. The deviation values at these locations are not representative of the true deviation in those sediment layers as the inversion procedure had very little impact on the estimates at these depths. It is also to be noted that in obtaining the deviation of the inverted parameters no prior variance has been assumed, and therefore the prior information does not constrain the solution. Tables III and IV also include values of resolution lengths estimated using Eq. (18). Although Eq. (18) provides the resolution length for each value of layer depth, these values were averaged over the thickness of the layer and are provided in Tables III and IV. We note that the resolution length has a small value in layer 1 and increases with the layer depth. This is because the mode function amplitude decays with depth, and hence the inverse results are less stable, resulting in the estimates being an average of values over a larger depth.

\section{DISCUSSION OF RESULTS}

\section{A. Comparison of experimentally measured mode dispersion with model predictions}

Figure 5 shows the spectrogram of the signals at waypoints 1,6 , and 8 compared with the mode dispersion as predicted by the models obtained by the inverse. Figure 5 shows good agreement between the data and model predictions based on the inverted model. Similar results were obtained in respect of mode dispersion data at other waypoints.

\section{B. Comparison with other geoacoustic models determined during the narrowband experiment}

The broadband experiment considered here was one component of MOMAX V. Another part consisted of a narrowband experiment in which Continuous Waveform (CW) tones at a set of frequencies were broadcast from a moving source. Two freely drifting buoys (Shemp and Larry) were deployed during this part of the experiment. The details of the experiment are explained in Ref. 3, and the tracks of the ship and the buoys are shown in Fig. 6. As seen in Fig. 6, the narrowband experiment was conducted in the general area covered by transmissions from waypoints 3 and 4 . The ship's track during the narrowband experiment, for the most part, lies in region II and is bounded by the source/receiver paths from waypoints 3 and 4 .

The bottom models obtained from the narrowband data ${ }^{3}$ and broadband data are compared in Table V. There are differences in the compressional wave speed estimates between those from the narrowband experiment and the broadband experiment. Some of these differences are attributable to the differences in layer thicknesses used in the inversions. Further, in the inversions done with the broadband data, the density of the sediment layers and the compressional wave speed of the density in the half space were also included as unknowns to be determined by the inversion. In the inversions done on the narrowband data, the density of the sediment layers and the half space were set at preset values. The same was the case with the compressional wave speed in the half space. With respect to the differences in the estimates for the third layer, the values from the broadband data are generally more consistent with the model obtained using data collected by Larry in the narrowband experiment but are significantly different from the values obtained using the Shemp data. To understand this discrepancy, we look at the differences in the data collected by Shemp and Larry during the narrowband experiment. The paths between the sources and receivers in the cases of the narrowband and broadband experiments are shown in Fig. 7. The ship's path during transmissions to Shemp and Larry are the same. The locations of the buoys are different, however, with Larry located to the south of Shemp. It is likely that the differences in the bottom models obtained from data collected by these two buoys are the result of the areas covered by the transmissions to these buoys. This suggests that the region to the

TABLE IV. Density deviations (Dev) and resolution lengths (RL) in the sediment for the five regions.

\begin{tabular}{|c|c|c|c|c|c|c|c|c|c|c|}
\hline \multirow[b]{2}{*}{ Layer } & \multicolumn{2}{|c|}{ Region I } & \multicolumn{2}{|c|}{ Region II } & \multicolumn{2}{|c|}{ Region III } & \multicolumn{2}{|c|}{ Region IV } & \multicolumn{2}{|c|}{ Region V } \\
\hline & $\operatorname{Dev}(\mathrm{gm} / \mathrm{cc})$ & $\mathrm{RL}(\mathrm{m})$ & $\operatorname{Dev}(\mathrm{gm} / \mathrm{cc})$ & $\mathrm{RL}(\mathrm{m})$ & $\operatorname{Dev}(\mathrm{gm} / \mathrm{cc})$ & $\mathrm{RL}(\mathrm{m})$ & $\operatorname{Dev}(\mathrm{gm} / \mathrm{cc})$ & $\mathrm{RL}(\mathrm{m})$ & $\operatorname{Dev}(\mathrm{gm} / \mathrm{cc})$ & $\mathrm{RL}(\mathrm{m})$ \\
\hline 1 & 0.0303 & 1.077 & 0.0228 & 1.104 & 0.115 & 1.084 & 0.065 & 1.15 & 0.038 & 1.11 \\
\hline 2 & 0.0187 & 1.557 & 0.0098 & 1.488 & 0.074 & 1.625 & 0.011 & 2.798 & 0.005 & 3.00 \\
\hline 3 & 0.0051 & 4.925 & 0.0037 & 4.742 & 0.019 & 6.285 & 0.003 & 16.127 & 0.002 & 15.49 \\
\hline
\end{tabular}



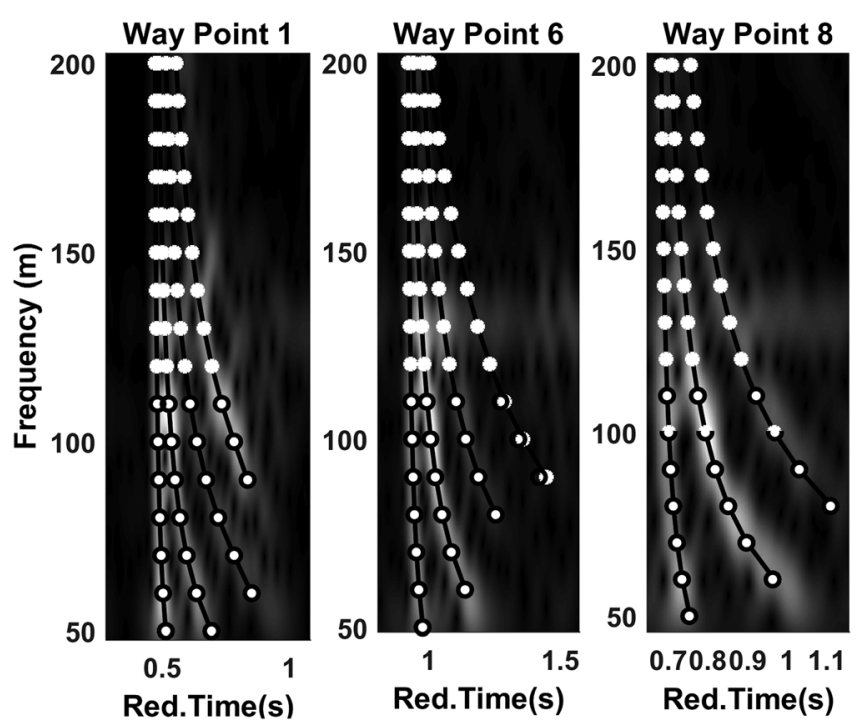

FIG. 5. Spectrograms of data received by Curly for transmissions from waypoints 1,6, and 8 . The mode dispersion curves estimated from the data are overlaid. The circles in the plots indicate the mode travel time as predicted by the inverted bottom model.

south of the areas covered by transmissions to Shemp have a slightly different acoustic property, especially with respect to the third layer. During the broadband experiment, the data used for inversions of regions I and II are from transmissions from waypoints 1,3 , and 4 . While the paths from waypoints 1 and 3 cover areas to the south of the paths during the narrowband experiment, the path from waypoint 4 covers an area to the north of the region covered in the narrowband experiment. However, the inverted profile for the sediment compressional wave speed for region II is similar to that obtained from narrowband data collected by Larry. This suggests that the inverted bottom model for region II is representative of the sediment in areas south of the paths covered during the narrowband experiment.

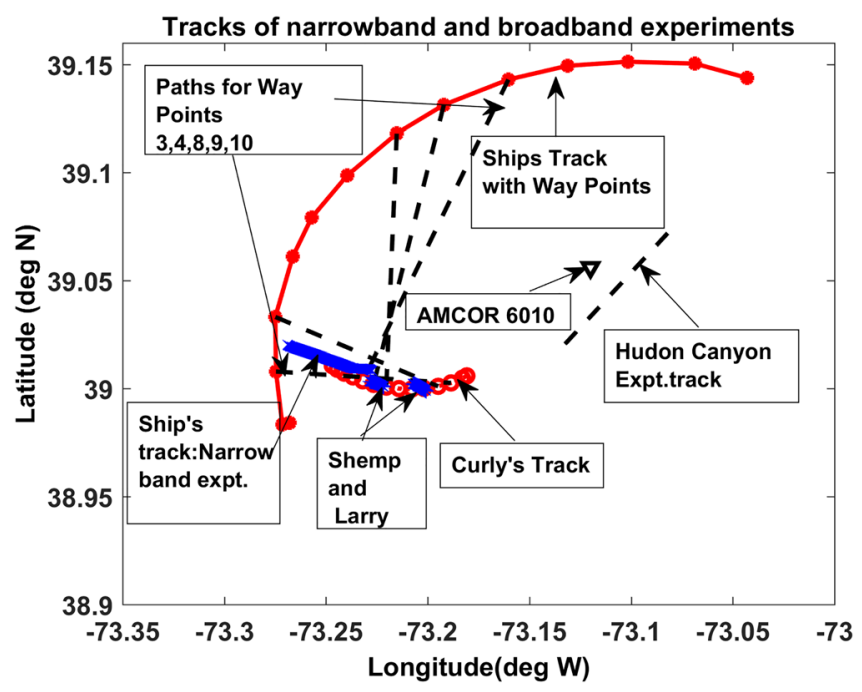

FIG. 6. (Color online) The ship and Curly tracks during the broadband experiment. The tracks of the ship and the buoys during the MOMAX V narrowband experiment are also indicated.
TABLE V. Inverted bottom models for region II from narrowband (NB) and broadband $(\mathrm{BB})$ experiments.

\begin{tabular}{lcccc}
\hline \hline Experiment & $\begin{array}{c}\text { Layer 1 } \\
\text { wave speed } \\
(\mathrm{m} / \mathrm{s})\end{array}$ & $\begin{array}{c}\text { Layer 2 } \\
\text { wave speed } \\
(\mathrm{m} / \mathrm{s})\end{array}$ & $\begin{array}{c}\text { Layer 3 } \\
\text { wave speed } \\
(\mathrm{m} / \mathrm{s})\end{array}$ & $\begin{array}{c}\text { Half space } \\
\text { wave speed } \\
(\mathrm{m} / \mathrm{s})\end{array}$ \\
\hline NB/Larry/SB795 & 1588 & 1702 & 1657 & 1850 \\
NB/Shemp & 1568 & 1705 & 1527 & 1850 \\
BB/Curly/region II & 1594 & 1661 & 1608 & 1863 \\
\hline \hline
\end{tabular}

We now try to predict the pressure fields measured in the narrowband experiment using the inverted bottom model for region II from the broadband experiment. In computing the pressure fields, all other parameters from the narrowband experiment, such as the water column sound speed profile, the range-dependent bottom depths, and the source and receiver depths, were used. The only differences were the geoacoustic parameters of the bottom. The pressure fields were computed at four frequencies, i.e., $50 \mathrm{~Hz}, 75 \mathrm{~Hz}, 125 \mathrm{~Hz}$, and $175 \mathrm{~Hz}$, using the normal mode code KRAKEN. ${ }^{20}$ These fields are plotted against the fields measured by Shemp and Larry during the experiment and are shown in Fig. 8.

With respect to the fields measured by Shemp and Larry during the narrowband experiment, we note that the agreement between the measured and predicted fields is better for Larry. It is also seen that the bottom models based on Larry data have a better agreement with the inverted profile from the broadband data as seen in Table V. To further assess agreement between the two results, we look at the corresponding wavenumber spectra. In Fig. 9, the wavenumber spectra obtained from the fields predicted by the broadband model are plotted against the wavenumber spectra obtained from the fields measured during the narrowband experiment. There is good agreement between the two in the case of strong lower order modes. The differences are pronounced in the case of higher order modes. This indicates that the dominant interference pattern, which is due to the lower order modes, will approximately be the same for both

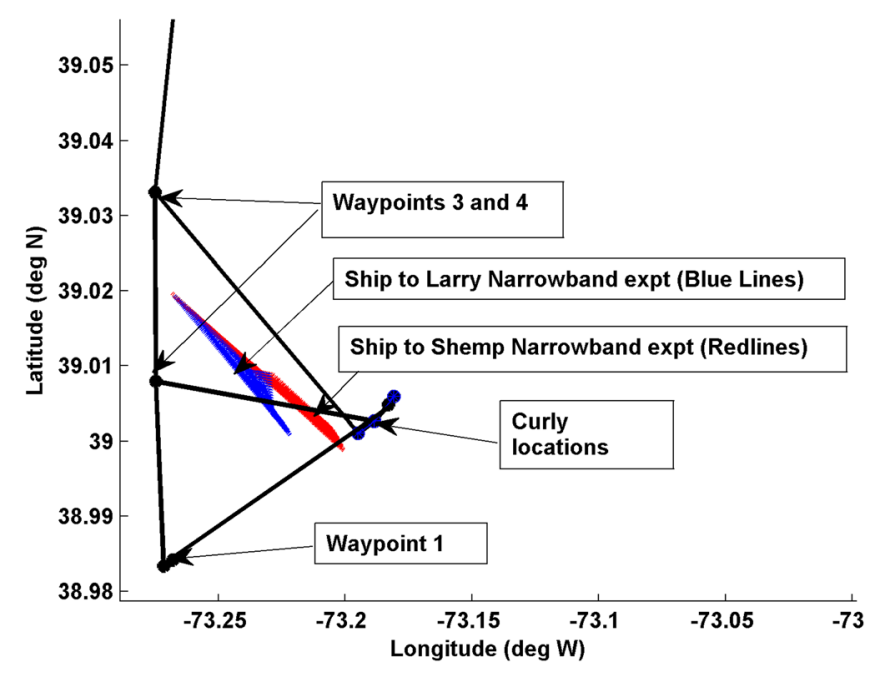

FIG. 7. (Color online) The paths between the sources and receivers in the cases of the narrowband and broadband experiments. 

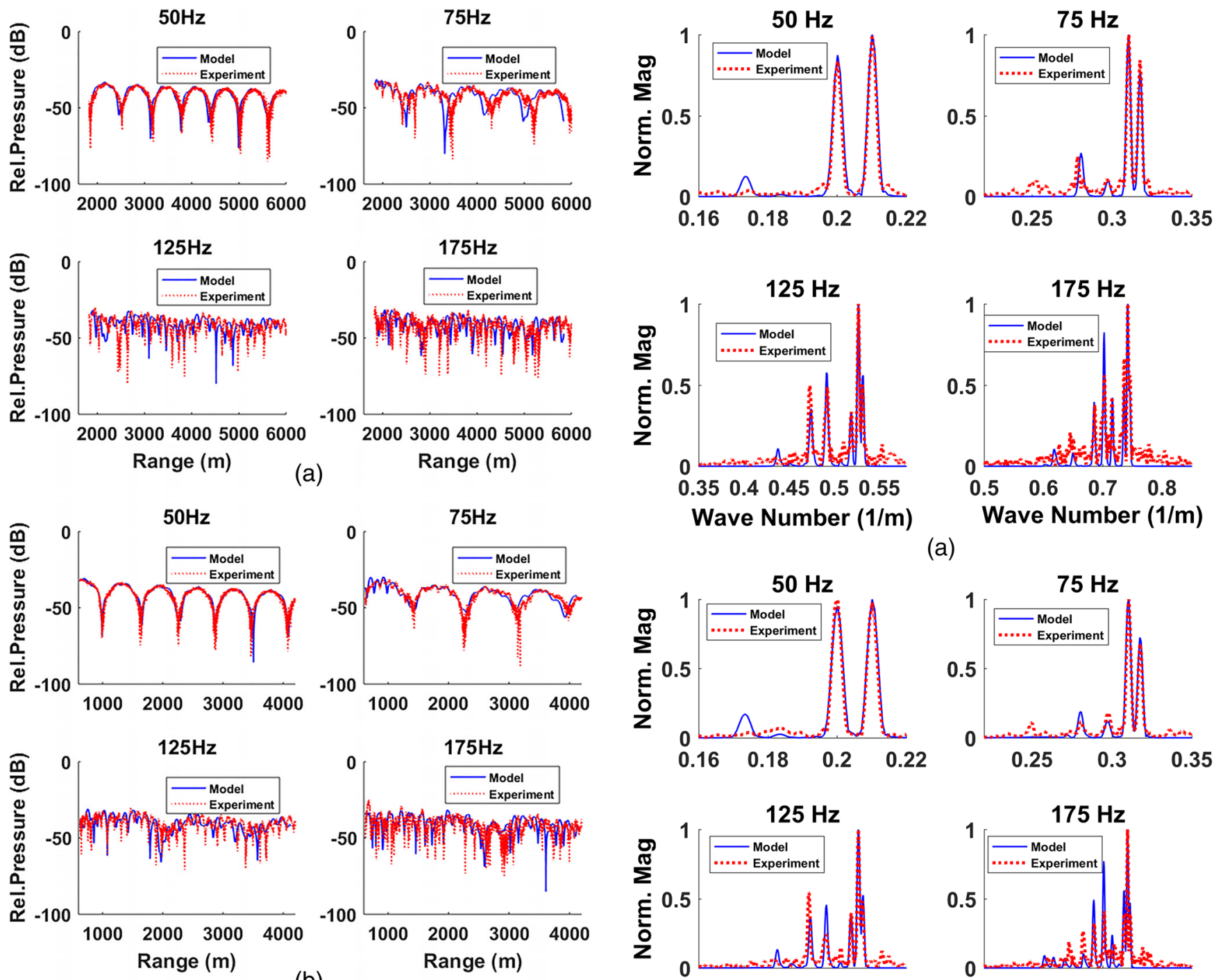

(b)
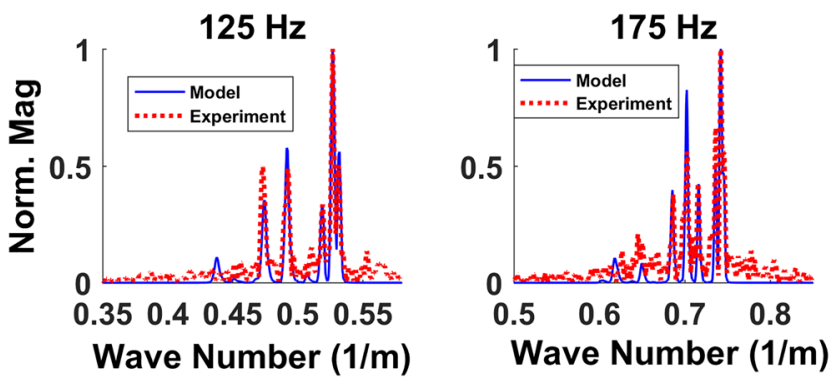

(a)
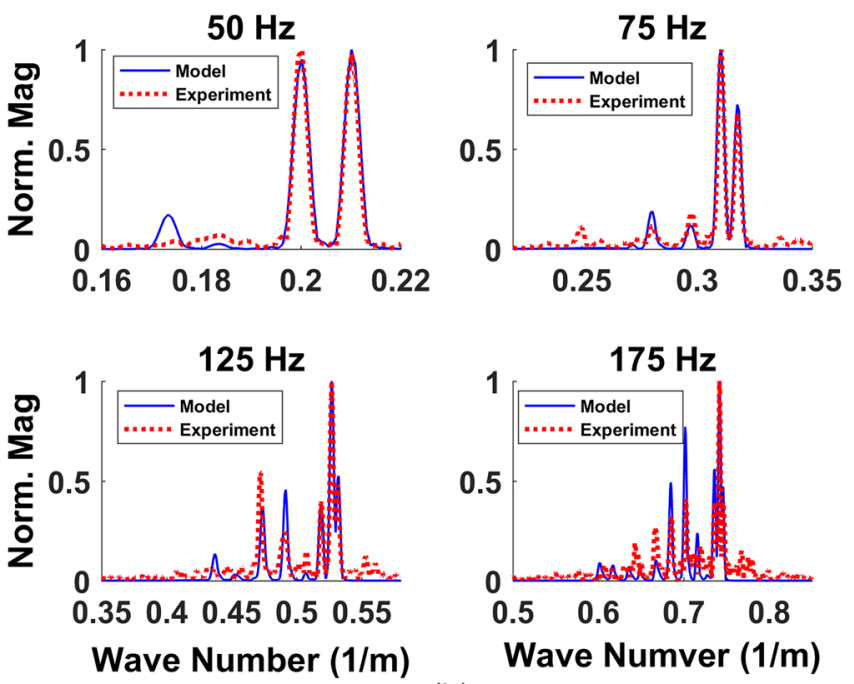

FIG. 8. (Color online) The pressure fields measured by buoys (Shemp and Larry) during the narrowband experiment and the fields predicted by the region II broadband model. The four plots in (a) compare the pressure fields measured at four different frequencies by Shemp and the fields predicted by the bottom model. The four plots in (b) compare the field measured by Larry with the model predictions.

cases. The differences in the wavenumber spectra of the higher order modes will manifest themselves as differences in the pressure fields that are superimposed upon the dominant interference pattern. This behavior is also observed in the plots shown in Fig. 8.

It is observed from Table I that the bottom models for regions IV and $\mathrm{V}$ are largely different from the values for other regions. Regions IV and $\mathrm{V}$ are located to the east of the other regions and are likely to represent a different set of bottom parameters. To verify the validity of these results, we compare the bottom model for region IV with the bottom model for an area close to AMCOR6010 in which the Hudson Canyon experiment ${ }^{21}$ (cf. Fig. 6) was conducted in 1988. The bottom model obtained from the Hudson Canyon experiment data and the bottom model for region IV are compared in the left panel of Fig. 10.

(b)

FIG. 9. (Color online) The wavenumber spectra obtained from the measured fields compared with the wavenumber spectra obtained from the predicted fields for Shemp (a) and Larry (b).

Figure 10 also compares the field measured during the experiment with the field predicted by the region IV model. Figure 10 shows fairly good agreement between the fields measured during the experiment and the field predicted by the region IV bottom model, thus validating results of inversion for the region.

\section{Comparison with other bottom models}

The sediment in the New Jersey shelf area can be classified as an amalgamation of sand, clay, sandy clay, and mud. ${ }^{16,17}$ The compressional wave speed values, density values, and their standard deviations for these types of sediments in the continental shelf areas are given in Table VI. ${ }^{22}$ The estimated compressional wave speed and density values for all of the five regions in Tables I and II fall within the range of values indicated in Table VI. 

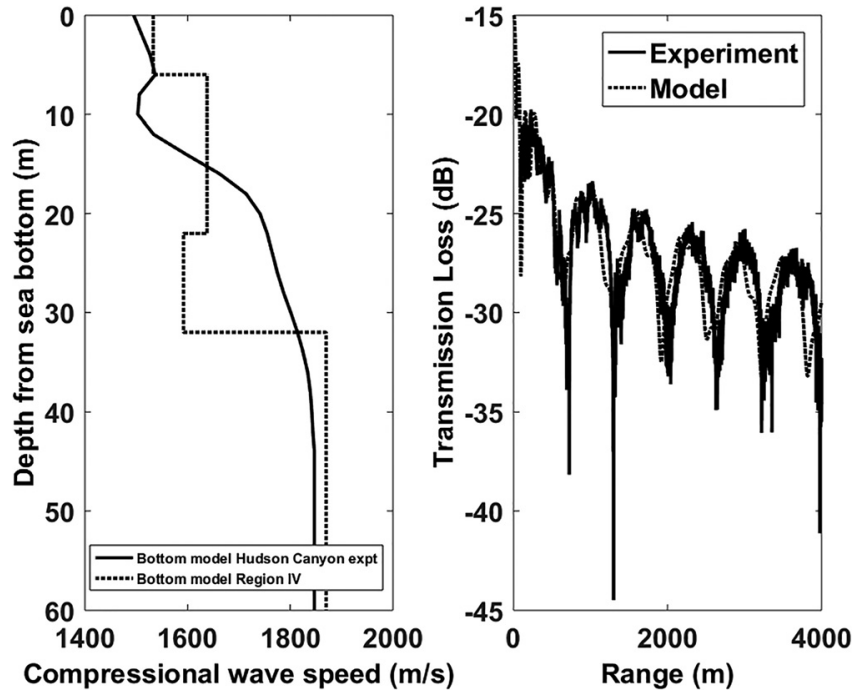

FIG. 10. The left panel shows the bottom model from the Hudson Canyon experiment (cf. Fig. 6) and the bottom model for region IV. The right panel shows the field measured during the experiment and the field predicted by the region IV bottom model.

The New Jersey shelf area has been the site of several acoustic experiments. The compressional wave speed results for the four experiments that took place in the general area of the SW06 experiment are summarized in Ref. 3 and reproduced here in Table VII. The locations of the experiments referred to in Table VII are shown in Fig. 11. The inner layered unit, the layer below the $\mathrm{R}$ reflector, and the deep low speed layer in Table VII correspond to layers 1-3 in Tables I and II, where estimates from the analysis of MOMAX V broadband data are tabulated. The values of the sediment compressional wave speeds for each sediment unit estimated using MOMAX V data are in general agreement with the compressional wave speed values as indicated in Ref. 3 and the speeds estimated during the SW06 experiment. ${ }^{2,10,23-25}$ Differences present in the estimated values from different experiments may be attributed to spatial variability within each unit, the parameterization of the
TABLE VI. Sediment type and their typical properties from Ref. 22.

\begin{tabular}{lcccc}
\hline \hline $\begin{array}{l}\text { Type of } \\
\text { sediment }\end{array}$ & $\begin{array}{c}\text { Compressional } \\
\text { wave speed (m/s) }\end{array}$ & $\begin{array}{c}\text { Standard } \\
\text { deviation }\end{array}$ & $\begin{array}{c}\text { Density } \\
(\mathrm{gm} / \mathrm{cc})\end{array}$ & $\begin{array}{r}\text { Standard } \\
\text { deviation }\end{array}$ \\
\hline Sand (fine) & 1749 & 11 & 1.941 & 0.023 \\
Sandy silt & 1652 & 12 & 1.771 & 0.033 \\
Clayey silt & 1549 & 4 & 1.488 & 0.016 \\
Sand-silt-clay & 1579 & 8 & 1.596 & 0.022 \\
\hline \hline
\end{tabular}

inversion technique, as well as the frequency band used in obtaining the results. The bottom models obtained in earlier SW06 experiments do not indicate the presence of a low speed layer below the $\mathrm{R}$ reflector, as is the case with the models from the MOMAX V experiment. However, analysis of data acquired near the AMCOR6010 site during an experiment in 1988 (Hudson Canyon experiment ${ }^{26,27}$ produced a bottom model with a low speed layer below the reflector. The data analyzed in this case were collected on transmissions along a long range-dependent track and are different from data analyzed in Ref. 21.

In the case of experiments conducted during SW06, only two experiments estimated the density values from experimental data. In other cases, the values were assumed based on some archival data or, in one case, based on porosity data. In the case of experiments that estimate the density values from the experimental data, there is general agreement between these estimated values and those predicted by MOMAX V.

\section{CONCLUSION}

In this paper, broadband data obtained during the MOMAX $\mathrm{V}$ experiment are processed and the data are inverted to simultaneously estimate the 3D compressional wave speed and density profiles of the seabed and sound speed profiles in the water column. The experimental data were acquired on a set of freely drifting, GPS-navigated buoys and a nearly stationary, low-frequency $(50-300 \mathrm{~Hz})$

TABLE VII. Summary of previous results of compressional wave speed values $(\mathrm{m} / \mathrm{s})$ and density $(\mathrm{gm} / \mathrm{cc})$. Results of the broadband experiment are indicated by BB. (Values of density marked with “*” were assumed values and not estimated from data; values marked with "\#” were calculated from porosity values from AMCOR6010 data. Density estimates with no marking were estimated from experimental data.)

\begin{tabular}{|c|c|c|c|c|c|c|c|c|c|}
\hline \multicolumn{2}{|l|}{ Property } & \multicolumn{4}{|c|}{ Compressional wave speed $(\mathrm{m} / \mathrm{s})$} & \multicolumn{4}{|c|}{ Density (gm/cc) } \\
\hline Reference & $\begin{array}{l}\text { Frequency } \\
(\mathrm{Hz})\end{array}$ & $\begin{array}{l}\text { Sand } \\
\text { ridge }\end{array}$ & $\begin{array}{c}\text { Diff. } \\
\text { reflective unit }\end{array}$ & $\begin{array}{c}\text { Inner layered } \\
\text { unit }\end{array}$ & $\begin{array}{l}\text { Below R } \\
\text { reflector }\end{array}$ & $\begin{array}{l}\text { Deep low- } \\
\text { speed layer }\end{array}$ & $\begin{array}{c}\text { Inner layered } \\
\text { unit }\end{array}$ & $\begin{array}{l}\text { Below } \mathrm{R} \\
\text { reflector }\end{array}$ & $\begin{array}{l}\text { Deep low- } \\
\text { speed layer }\end{array}$ \\
\hline Ballard et al. (Ref. 10) & $125-175$ & & $1670 \pm 12$ & $1580 \pm 19$ & $1725 \pm 15$ & & $1.9^{*}$ & $1.9^{*}$ & \\
\hline Rajan and Becker (Ref. 2) & $30-120$ & & $1660-1680$ & $1510-1650$ & $1650-1850$ & & $1.6^{*}$ & $1.6^{*}$ & \\
\hline Jiang and Chapman (Ref. 23) & $50-1000$ & & $1636 \pm 15$ & $1572 \pm 15$ & $1740 \pm 40$ & & 1.68 & 1.68 & \\
\hline Knobles et al. (Refs. 24, 25) & $35-265$ & $1650-1700$ & & $1580-1595$ & 1720 & & 1.83 & 1.93 & \\
\hline Cederberg et al. (Ref. 26) & $50-75$ & $1560-1640$ & & $1510-1550$ & $1770-1790$ & 1670 & $1.9^{\#}$ & $2.1^{\#}$ & $2.2^{\#}$ \\
\hline \multicolumn{10}{|l|}{ Cederberg et al. (Ref. 27) } \\
\hline $\mathrm{BB} /$ region I & $50-110$ & & & 1569 & 1644 & 1604 & 1.602 & 1.727 & 1.612 \\
\hline $\mathrm{BB} /$ region II & $50-110$ & & & 1594 & 1661 & 1609 & 1.520 & 1.638 & 1.600 \\
\hline $\mathrm{BB} /$ region III & $50-110$ & & & 1579 & 1687 & 1624 & 1.681 & 1.694 & 1.604 \\
\hline $\mathrm{BB} /$ region IV & $50-110$ & & & 1533 & 1638 & 1592 & 1.786 & 1.704 & 1.596 \\
\hline $\mathrm{BB} /$ region V & $50-110$ & & & 1506 & 1623 & 1593 & 1.549 & 1.708 & 1.597 \\
\hline
\end{tabular}




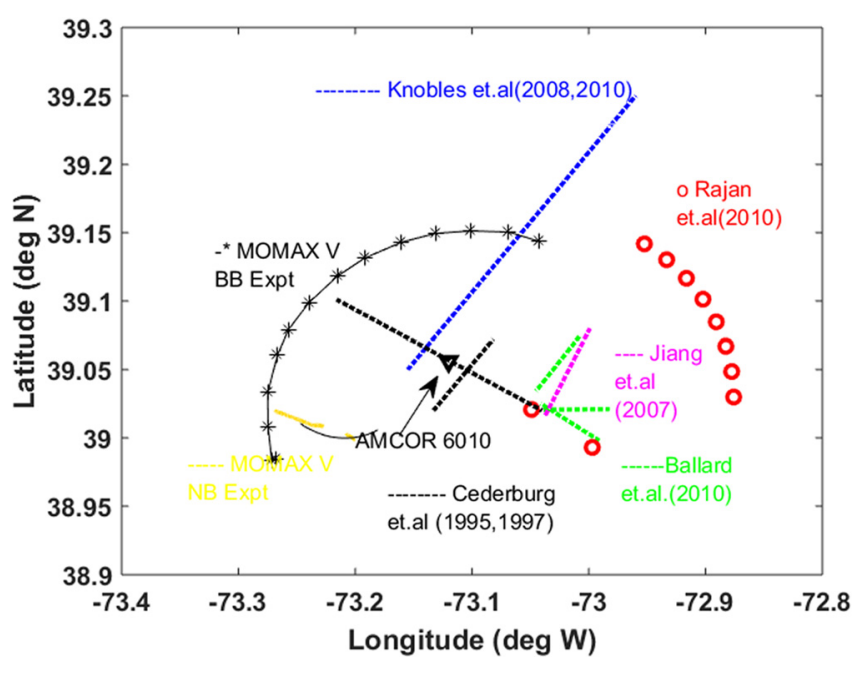

FIG. 11. (Color online) Locations of the experiments listed in Table VII.

sound source at a set of fixed locations. Bottom models for sediment properties over a wide geographical area were estimated using a linearized inversion method. The results are validated by comparing them with the bottom models obtained from the narrowband data acquired during the same experiment. Further validation was done by comparing the pressure fields computed using the broadband bottom models with the fields measured during the narrowband experiment. Also, the bottom models from the broadband experiment were compared with models obtained from other experiments conducted in the general area, and it was shown that the models are consistent with one another. This inversion method comes with its known limitations such as use of some prior information on the parameters being estimated so that a linear inversion results in acceptable values for the unknown parameters. The estimation of uncertainties of the parameters is also limited by the procedure used. Nevertheless, the results from the linearized inversion technique presented here show that the broadband data collected by a distributed network of buoys can be used to obtain reasonable estimates of both the 3D water column and sediment properties over a wide area.

\section{ACKNOWLEDGMENTS}

The authors gratefully recognize the support of the entire team that was responsible for the execution of MOMAX V. The work reported in this paper was done with the support of the Office of Naval Research under Grant Nos. N00014-09-1-0505 and N00014-12-1-0083 and the Space and Naval Warfare Systems Command through Naval Sea Systems Command Contract No. N00024-02-D-6604.

${ }^{1}$ S. D. Rajan, G. V. Frisk, K. M. Becker, J. F. Lynch, G. Potty, and J. H. Miller, "Modal inverse techniques for inferring geoacoustic properties in shallow water," in Important Elements in Geoacoustic Inversion, Signal Processing, and Reverberation in Underwater Acoustics, edited by A. Tolstoy (Research Signpost, Kerala, India, 2008), pp. 165-234.

${ }^{2}$ S. D. Rajan and K. M. Becker, "Inversion for range-dependent sediment compressional-wave-speed profiles from modal dispersion data," IEEE J. Ocean. Eng. 35, 43-58 (2010).
${ }^{3}$ G. V. Frisk, K. M. Becker, S. D. Rajan, C. J. Sellers, K. von der Heydt, C. M. Smith, and M. S. Ballard, "Modal mapping experiment and geoacoustic inversion using sonobuoys," IEEE J. Ocean. Eng. 40, 607-620 (2015). ${ }^{4}$ M. S. Ballard, G. V. Frisk, and K. M. Becker, "Estimates of temporal and spatial variability of ocean sound speed on the New Jersey shelf," J. Acoust. Soc. Am. 135, 3316-3326 (2014).

${ }^{5}$ G. Potty, J. H. Miller, J. F. Lynch, and K. Smith, "Tomographic imaging of sediment parameters in shallow water," J. Acoust. Soc. Am. 108, 973-986 (2000).

${ }^{6}$ G. Potty, J. Miller, and J. Lynch, "Inversion for sediment geoacoustic properties at the New England Blight," J. Acoust. Soc. Am. 114(4), 1874-1887 (2003).

${ }^{7}$ J. Bonnel, S. E. Dosso, and N. R. Chapman, "Bayesian geoacoustic inversion of single hydrophone light bulb data using warping dispersion analysis," J. Acoust. Soc. Am. 134(1), 120-130 (2013).

${ }^{8}$ S. D. Rajan, "Simultaneous reconstruction of compressional wave speed and density profiles from modal eigenvalues," J. Comput. Acoust. 6(1\&2), 257-267 (1994).

${ }^{9}$ L.Simcik and P. Lin, "Qualitative regularization: Resolving non-smooth solutions," Tech Report CSE 94-12, University of California, Department of Mathematics (1994).

${ }^{10}$ M. S. Ballard, K. M. Becker, and J. A. Goff, "Geoacoustic inversion for the New Jersey shelf: Three dimensional sediment model," IEEE J. Ocean. Eng. 35, 28-42 (2010).

${ }^{11}$ A. N. Tikhonov and V. Y. Arsenin, Solution of Ill-Posed Problems (Hallsted, Washington, D.C., 1977).

${ }^{12}$ M. S. Ballard and K. M. Becker, "Optimized constraints for the linearized geoacoustic inverse problem," J. Acoust. Soc. Am. 129(2), 652-661 (2011).

${ }^{13} \mathrm{~K}$. Aki and P. G. Richards, Quantitative Seismology Theory and Methods Vol II (Freeman, San Francisco, 1980).

${ }^{14}$ J. Bonnel, B. Nicholas, J. Mars, and S. C. Walker, "Estimation of modal group velocities with a single receiver for geoacoustic inversion in shallow water," J. Acoust. Soc. Am. 128, 719-727 (2010).

${ }^{15}$ J. Bonnel, C. Gervaise, P. Roux, B. Nicolas, and J. Mars, "Modal depth function estimation using time-frequency analysis," J. Acoust. Soc. Am. 130, 61-71 (2011).

${ }^{16}$ T. A. Davies, J. A. Austin, Jr., H. B. Lagoe, and J. D. Millimann, "Late quaternary sedimentation off New Jersey shelf: New results using 3-D seismic profiles and cores," Mar. Geol. 108, 323-343 (1992).

${ }^{17}$ J. A. Goff, B. A. Kraft, L. A. Mayer, S. G. Schock, C. K. Sommerfield, H. C. Olson, S. P. Gulick, and S. Nordfjord, "Seabed characterization on the New Jersey middle and outer shelf: Correlatability and spatial variability of seafloor sediment properties," Mar. Geol. 209, 147-172 (2004).

${ }^{18}$ D. J. Tang, J. Moum, J. Lynch, P. Abbot, R. Chapman, P. Dahl, T. Duda, G. Gawarkiewicz, S. Glenn, J. Goff, H. Graber, J. Kemp, A. Maffei, J. Nash, and A. Newhall, "Shallow Water '06 - A joint acoustic propagation/nonlinear internal wave physics experiment," Oceanography 20, 156-167 (2007).

${ }^{19} \mathrm{M}$. Ballard (private communication, 2012).

${ }^{20}$ M. Porter, "The Kraken normal mode program," Tech Report SM245, SACLANT Undersea Research Center, La Spezia, Italy (1991).

${ }^{21}$ S. D. Rajan, J. A. Doutt, and W. Carey, "Inversion for the compressional wave speed profile of the bottom from synthetic aperture experiments conducted in the Hudson Canyon area," IEEE J. Ocean. Eng. 23, 174-187 (1998).

${ }^{22}$ E. L. Hamilton, "Geoacoustical modeling of the sea floor," J. Acoust. Soc. Am. 68(5), 1313-1340 (1980).

${ }^{23}$ Y. M. Jiang and N. R. Chapman, "Bayesian geoacoustic inversion in a dynamic shallow water environment," J. Acoust. Soc. Am. 123, EL155-EL161 (2008).

${ }^{24}$ D. P. Knobles, J. A. Goff, R. A. Koch, P. S. Wilson, and J. A. Shooter, "Effect of inhomogeneous sub-bottom layering on broadband acoustic propagation," IEEE J. Ocean. Eng. 35, 732-743 (2010).

${ }^{25}$ D. P. Knobles, P. S. Wilson, J. A. Goff, and S. E. Cho, "Seabed acoustics of a sand ridge on the New Jersey continental shelf," J. Acoust. Soc. Am. 124, EL151-EL156 (2008).

${ }^{26}$ R. J. Cederberg, W. L. Siegmann, and W. M. Carey, "Influence of geoacoustic modeling on predictability of low-frequency propagation in range-dependent, shallow-water environments," J. Acoust. Soc. Am. 97, 2754-2766 (1995).

${ }^{27}$ R. J. Cederberg, W. M. Carey, and W. L. Siegmann, "Modal analysis of geoacoustic influences on shallow water propagation," IEEE J. Ocean. Eng. 22, 237-244 (1997). 